\title{
Random Pilot Signal Design and Channel Estimation Method based on Compressed Sensing Theory
}

\author{
$\mathrm{Li} \mathrm{Li}$ and Ning Chen \\ College of Computer Science, Xi'an Polytechnic University \\ chennvictor@gmail.com
}

\begin{abstract}
A channel parameter estimation method adapted to a wireless communication system is proposed. The wireless communication system includes a transmitter and a receiver. The proposed method includes following steps. The transmitter transmits a plurality of pilot signals to the receiver by using one of a plurality of preconfigured sparse random pilot patterns. The receiver receives the pilot signals, performs a channel parameter estimation on the pilot signals by using a compressive sensing algorithm to obtain a multipath channel number, and selects a pilot pattern for a next cycle among the preconfigured sparse random pilot patterns according to the multipath channel number and a current pilot number. Additionally, the receiver transmits feedback information associated with the selected pilot pattern to the transmitter.
\end{abstract}

Keywords: Pulse-Shaping Multicarrier,Compressive Sensing,Channel Estimation, A Random Pilot Signal

\section{Introduction}

Wireless communication technology begins to receive a lot of attention and also plays a very important role in our lives after overcoming the mobile problem. With the increasing of multimedia information and a variety of different high quality services, realtime communication demand between people is also showing exponential growth.

In a wireless communication system, to estimate channel response precisely to recover transmitted signal has become a very important research direction, in which the pilot symbol-aided channel estimation is the most common method to obtain channel information. Conventional channel estimation methods, for example, least squares(LS), least mean-square error(MMSE), etc., are assumed that the wireless transmission channel is dense multipath channel. More pilot symbols should be put into transmitted signal relatively to obtain more accurate channel responses, resulting in reduction in transmission rate. With further research, more and more tests show that the radio channel has the sparse feature. Namely, less than about $10 \%$ of the multipath channels occupy more than $85 \%$ of the channel energy[1]. As long as finding out and taking advantage of the sparse feature in channel, the number of pilot symbols can be reduced greatly, and the spectrum utilization can also be improved effectively.

In recent years, Candes, Romberg[2], Tao[3], Donobo[4] and other scientists have proposed a new information theory, namely compressed sensing. The theory indicates that: when the signal is sparse or compressible in a transform domain, measurement matrix irrelevant to transformation matrix can be used to project transform coefficients to low-dimensional measurement matrix linearly maintaining the information needed to reconstruct the signal. The original high-dimensional signal can be reconstructed from low-dimensional measurement matrix accurately or with high probability accurately by further solving sparse optimization problem. Compressed sensing theory is used in many fields, such as image processing, medical imaging, pattern recognition, geological exploration, optics, radar imaging, wireless communication and other aspects. 
This paper focuses on applying compressed sensing theory to channel estimation, using the Doppler sparsity in channel delay and matching tracking algorithm based on the model of multi-carrier system[5], to obtain accurate channel information through less pilot signals for the sake of improvement on the spectrum utilization.

\section{Compressed Sensing Theory}

In recent years, compressed sensing theory has been widely used in the fields of applied mathematics and signal processing. It provides a theoretical arthitecture and efficient algorithms to reconstruct the original sparse signal with less measurement signals. More speciffically, compressed sensing is to estimate the sparse reconstruction problem of an unknown sparse vector $u$ from the observation vector $v$ that can be measured. While the observation vector $v$ is a $N \times 1$ dimensional row vector in $R^{N}$ space, the sparse vector $u$ is a $M \times 1$ dimensional row vector in $R^{M}$ space. The mathematical expression is as follow:

$$
\mathbf{v}=\boldsymbol{\Phi} \mathbf{u}+\mathbf{w}
$$

In the mathematical expression, $\boldsymbol{\Phi}_{\text {is a }} N \times M$ dimensional measurement matrix, $\mathbf{w}$ is a $N \times 1$ dimensional noise vector. The restriction in the reconstruction is assuming $\mathbf{u}$ is $\mathrm{S}$ sparse, in which there are at least $S$ non-zero inputs and their positions are unknown. Generally speaking, the number of unknown data to be measured is much larger than the known, such as $M \gg N$.

The key factor of compressed sensing is uniform uncertainty principle[6], which essentially illustrates that the measurement matrix $\boldsymbol{\Phi}$ satisfies the "restricted isometry property"(RIP) condition. Assume that the row of $\boldsymbol{\Phi}$ is composed of sub-matrix $\boldsymbol{\Phi}_{\mathrm{r}}$ and take the elements of $T$ as index. $\Phi_{\mathrm{r}}$ is a $N \times|T|$ sub-matrix. Then, define $S_{\text {restricted }}$ isometry constant $\delta_{S}$ of the measurement matrix $\boldsymbol{\Phi}$ as the smallest positive number ${ }^{\delta_{s}}$ :

$$
\left(1-\delta_{s}\right)\|\alpha\|_{2}^{2} \leq\left\|\Phi_{\tau} \alpha\right\|_{2}^{2} \leq\left(1+\delta_{s}\right)\|\alpha\|_{2}^{2}
$$

All the $\mathrm{S}$-sparse matrices $\mathbf{a}$ are $M \times 1$ dimensional row vectors in $R^{M}$ space.

For the solving of estimator $\hat{\mathbf{u}}$ of $\mathbf{u}$, we can combine the characteristic of sparse signal and find the accurate or approximate value of coefficient $\mathbf{u}$ based on ${ }^{l_{1}}$-norm(norm) minimization. The expression is defined as follow:

$$
\hat{\mathbf{u}}=\arg \min _{u \in u_{\varepsilon}}\|\mathbf{u}\|_{1}
$$

In the equation, \|\|$_{k}$ represents the norm of $k_{\text {-stage, while }} v_{\varepsilon}$ is the set of all $u \in R^{M}$. When $\varepsilon>0$ is given, the condition $\square \boldsymbol{\Phi u}-\mathbf{v} \square_{2} \leq \varepsilon$ must be met. According to the result of the following theorem, the estimator $\hat{\mathbf{u}}$ can restore parameter vector of $S$-sparse.

Now given a $S$, and assum $3 S$ and $4 S-R I P$ of $\boldsymbol{\Phi}$ satisfy the following formula:

$$
\delta_{3 S}+3 \delta_{4 S}<2
$$

Suppose $\mathbf{v}=\boldsymbol{\Phi} \mathbf{u}+\mathbf{w}$ and $\square \mathbf{w} \square_{2} \leq \varepsilon$, and assume $\mathbf{u}_{S}$ is a $M \times 1$ dimensional row vector in $R^{M}$ space, which contains $S$ elements of maximum absolute value within $\mathbf{u}$, while the remaining $M-S$ elements are zero. Therefore, the estimator $\hat{\mathbf{u}}$ in equation (3) will meet the following condition:

$$
\|\hat{\mathbf{u}}-\mathbf{u}\|_{2} \leq C_{1} \varepsilon+C_{2} \frac{\left\|\mathbf{u}-\mathbf{u}_{\mathbf{s}}\right\|_{1}}{\sqrt{S}}
$$


The constants $C_{1}$ and $C_{2}$ in the equation depend on $\delta_{35}$ and $\delta_{45}$.

For a relatively independent distributed Gaussian noise vector with expectation value 0 , there is a high probability to find a suitable $\varepsilon$ to satisfy the condition $\|\mathbf{w}\|_{2} \leq \varepsilon$. In the case of no noise, for a ${ }^{S-\text { sparse }}$ vector $\mathbf{u}$, the equation (5) can be rewritten as $\hat{\mathbf{u}}=\mathbf{u}$.

The case that measurement matrix $\boldsymbol{\Phi}$ satisfy the equation (4) is a research focus currently.

Assume that $N^{\prime}$ columns are selected uniformly and randomly to merge rows from a $M^{\prime} \times M^{\prime}$ unitary matrix $U$ to realize a normalization. Therefore, adopting the unit Euclidean norm and using these rows to reconstruct a complex measurement matrix $\boldsymbol{\Phi}_{c} \in C^{N \times M^{\prime}}$, there is a great probability to make equation (4) be established. The lower bound of the following observation data's number can provide a sufficient condition[2] [6] [7].

$$
N^{\prime} \geq C_{3}\left(\ln M^{\prime}\right)^{4} \mu^{2} S
$$

In the expression, $\mu$ is the coherence of $U$ and defined as $\sqrt{M^{1}} \max _{i, j}\left|U_{i, j}\right|$, while $C_{3}$ is a constant item. Then we have the same great probability to satisfy equation (4) in the case of equation (6), namely the real number's measurement matrix $\boldsymbol{\Phi} \in R^{N \times M}$ (wherein $\left.N=2 N^{\prime}, M=2 M^{\prime}\right)$ of $\boldsymbol{\Phi}_{C}$ can be calculated on the basis of the following equation.

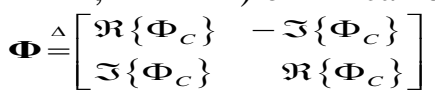

\section{Channel Estimation}

\subsection{Multi-carrier System Model}

Since the advantage of pulse shaping multi-carrier system surpasses traditional cyclic prefix orthogonal frequency division multiplexing system(CP-OFDM) [8] [9], here we consider pulse shaping multi-carrier system, and always consider the complex baseband domain. Suppose $K, N \geq K$ and $L$ represent the number of subcarries, the symbol range and the periods number of transmitted symbols. The discrete transmitted symbols of multi-carrier are as follows:

$$
s[n]=\sum_{l=0}^{L-1} \sum_{k=0}^{K-1} a_{l, k} g_{l, k}[n]
$$

Thereinto, $a_{l, k}$ is data symbol, while ${ }^{g_{l, k} \stackrel{\Delta}{=} g[n-l N] e^{j 2 \pi \frac{k}{K}(n-l N)}}$ is the time of transmitted pulse minus the offset changes of frequency. Then convert $s[n]$ to continuous-time transmitted signal.

$s(t)=\sum_{n=-\infty}^{\infty} s[n] f\left(t-n T_{s}\right)$

Thereinto, $f(t)$ is a interpolation filter, while $T_{S}$ is the sampling period. For simplicity, we assume an ideal filter, namely $f(t)=\sqrt{1 / T_{S}} \sin c\left(\pi t / T_{S}\right)$.

Assume that the channel is a selective dispersion time-varying pulse response $h(t, \tau)$, the signal received by receiver is as follow:

$$
r(t)=\int_{-\infty}^{\infty} h(t, \tau) s(t-\tau) d \tau+z(t)
$$

In the equation, ${ }^{z(t)}$ is a complex white Gaussian noise with expectation value 0 and power spectral density ${ }^{N_{0}}$. Then convert the signal into discrete signal. The mathematical formula is:

$$
r[n]=\int_{-\infty}^{\infty} r(t) f\left(t-n T_{S}\right) d t
$$


Thereinto, $f(t)$ can be viewed as anti-aliasing filter. The demodulated signal calculated by multi-carrier demodulator is:

$$
x_{l k}=\left\langle r, \gamma_{l, k}\right\rangle=\sum_{-\infty}^{\infty} r[n] \gamma_{l, k}^{*}[n]
$$

We can obtain the relationship between discrete channel ${ }^{s[n]}$ and discrete signal $r[n]$ after combination of above equations.

$$
r[n]=\sum_{m=-\infty}^{\infty} h[n, m] s[n-m]+z[n]
$$

Thereinto, discrete time-varying pulse response is:

$$
h[n, m]=\int_{-\infty}^{\infty} h\left(n T_{s}, \tau\right) \sin c\left(\pi\left(m-\frac{\tau}{T_{s}}\right)\right) d \tau
$$

According to equation (8), (12) and (13), and ignoring the interference between intersymbol and inter-subcattier, we can obtain the expression of delayed Doppler domain:

$$
r[n]=\sum_{m=-\infty}^{\infty} \sum_{i=0}^{N-1} S_{h}[m, i][n-m] e^{j 2 \pi \frac{i n}{N_{t}}}+z[n]
$$

Thereinto, the discrete delayed Doppler spread function[10] is defined as follow:

$$
S_{h}[m, i] \triangleq \frac{1}{N} \sum_{n=0}^{N=1} h[n, m] e^{-j 2 \pi \frac{i n}{N_{r}}}
$$

Through equation (8), (12) and (15), we have:

$$
H_{l, k}=\sum_{m=\infty}^{\infty} \sum_{i=0}^{N-1} F[m, i] e^{-j 2 \pi\left(\frac{(m m}{K} \cdot \frac{N L I}{N_{r}}\right)}
$$

Thereinto, cross fuzzy function[11]is defined as follow:

$$
A_{r, g(m, \xi)} \stackrel{\Delta}{=} \sum_{m=-\infty}^{\infty} \gamma[n] g^{*}[n-m] e^{-j 2 \pi \xi^{n} n}
$$

Since $N_{r}$ and $L N$ are similar, equation (16) can be rewritten to a two-dimensional discrete Fourier transform, and its mathematical formula is as follow:

$$
H_{l, k}=\sum_{m=0}^{K-1} \sum_{i=0}^{L-1} \tilde{F}[m, i] e^{-j 22\left(\frac{k m-l}{K}-\frac{i t}{L}\right)}
$$

Thereinto, $\bar{F}[m, i]$ is defined as follow:

$$
\tilde{F}[m, i]=\sum_{q=0}^{\Delta N-1} F[m, i+q L], i=0, \ldots, L-1
$$

\subsection{Delayed Doppler Sparsity}

We assume that the channel is composed of $P$ scattering paths with a fixed delay $V_{p}$ and Doppler frequency offset $p=1, \cdots, P$. Its channel pulse response can be represented as:

$$
h(t, \tau)=\sum_{p=1}^{p} \eta_{p} \delta\left(\tau-\tau_{p}\right) e^{j 2 \pi v_{p}, t}
$$

Thereinto, $\eta_{p}$ is the decay and initial phase of the first $p$ path. The discrete form of equation (20) can be represented as:

$$
h[n, m]=\sum_{p=1}^{p} \eta_{p} e^{j 2 \pi r_{p}, r_{s}} \sin c\left(\pi\left(m-\frac{\tau_{p}}{T_{s}}\right)\right)
$$

Substitute equation (22) into equation (16), then we can obtain the discrete delayed Doppler spread function. Its mathematical equation is as follow:

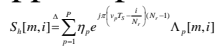

Thereinto, the function ${ }^{\Lambda_{p}}$ is defined as follow:

$$
\begin{aligned}
& \Lambda_{p}[m, i]=\sin c\left(\pi\left(m-\frac{T_{p}}{T_{s}}\right)\right) \operatorname{dir}\left(\pi\left(i-v_{p} T_{s} N_{r}\right)\right)
\end{aligned}
$$

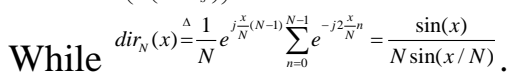




\subsection{Channel Estimation based on Compressed Sensing}

Now we combine PSACE and CS. Transmitted and received pulses are includeded in the actual channel, and the function ${ }^{F[m, i]}$ in equation (18) can provide a sub-domain in delayed Doppler plane effectively, so we can do subsampling in the time-frequency domain. Suppose function $F[m, i]$ is contained in $[0, D-1] \times([0, I / 2-1] \cup[L-I / 2, L-1])$, thereinto, $D \leq K, I$ is an even number. For the selection of $D$ and $I$, both $A K \square K / D$ and $\Delta L \square L / I$ must be integer value. Therefore, the channel coefficient can be described as the value of subsampling $(l, k)=\left(l^{\prime} \Delta L, k^{\prime} \Delta K\right)$ specifically based on equation (19). So equation (19) can be rewritten as:

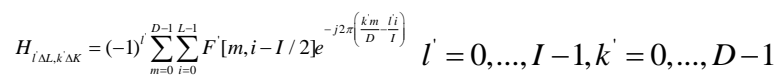

Thereinto, $F^{\prime}[m, i]$ is the periodically expanded form, such as $\tilde{F}[m, i+L]$.

Assume that pilot symbol $a_{l, k}=p_{l, k}$ is sent in the position of time-frequency grid $(l, k) \in P$,

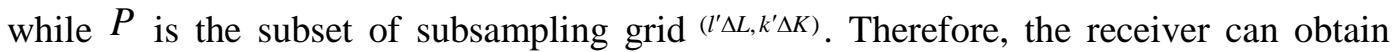
channel coefficient through equation (25).

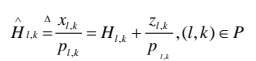

In the equation (24), every $\mathrm{H}_{l^{\prime} \Delta L, k^{\prime} \Delta k}$ can be represented by Fourier transform relationship. The vector expression of equation (24) is as follow:

$$
\mathbf{h}=\sqrt{l D} \mathbf{U} \mathbf{u}_{\mathbf{c}}
$$

Here we define: (1) $I D$ dimensional complex parameter vector, (2) $I D$ dimensional complex channel vector. Their mathematical equations are as follows:

$$
\begin{aligned}
& \left.\mathbf{u}_{\mathrm{c}}=\mathbf{u}_{c}^{T}(0) \ldots \mathbf{u}_{c}^{T}(D-1)\right]^{T} \\
& \stackrel{\Delta}{\mathbf{h}}=[\mathbf{h}(0) \ldots \mathbf{h}(D-1)]
\end{aligned}
$$

Thereinto,

$$
\begin{aligned}
& \mathbf{u}_{c}(m) \stackrel{\Delta}{\stackrel{\Delta}{*}\left[F^{\prime}[m,-I / 2] \ldots F^{\prime}[m,-I / 2-1]\right]}
\end{aligned}
$$

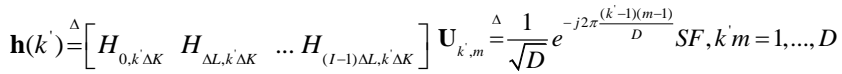

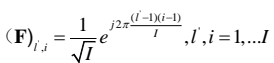

$S_{\text {is a }} I \times I$ diagonal matrix, its constituent elements are ${ }^{1,-1,1,-1, \cdots}$. We also define a $I D \times I D$ frame matrix $\mathbf{U}$ consisted of $I \times I$ sized block $\mathbf{U}_{k^{\prime}, m}$.

Channel vector can obtain channel coefficient ${ }^{H}{ }_{l, k}$ in the position of $(l, k) \in P$ via pilot symbol ${ }^{|P|}$. Suppose $\mathbf{h}^{(p)}$ is a ${ }^{|P|}$ dimensional subvector in $\mathbf{h}_{\text {and }} \boldsymbol{\Phi}_{c}$ is a $|P| \times I D$ sub-matrix consisted of ${ }^{|P|}$ columns in $\mathbf{U}$. We can get a measurement equation after reorganization and subsitution:

$$
\mathbf{v}=\boldsymbol{\Phi} \mathbf{u}+\mathbf{W}
$$

We can use CS to estimate the channel parameters and create measurement matrix on the basis of these parameters from the above theory. While the non-zero elements can be mapped to the position of pilot symbol in transmitted signal. Then, convert equation (29) to the following mathematical expression: 


$$
\left[\begin{array}{c}
H_{0} \\
H_{1} \\
\vdots \\
H_{m-2} \\
H_{m-1}
\end{array}\right]_{m \times 1}=\mathbf{A}_{m \times N} \cdot \mathbf{B}_{N \times N} \cdot\left[\begin{array}{c}
f_{1}^{\prime} \\
0 \\
\vdots \\
0 \\
f_{2}^{\prime} \\
0 \\
\vdots \\
0
\end{array}\right]_{N \times 1}+\left[\begin{array}{c}
W_{0} \\
W_{1} \\
\vdots \\
W_{m-2} \\
W_{m-1}
\end{array}\right]_{m \times 1}
$$

Thereinto, $\mathbf{B}$ is a two-dimensional fast Fourier transformation matrix, $\mathbf{A}$ is a selection matrix used to select specific columns from $\mathbf{B}$ to run CS. $m$ represents the number of pilot symbols. The sender and receiver can obtain $m$ through the fast Fourier transformation matrix and selection matrix before communication.

Now we consider a multi-carrier system. Figure 1 shows system architecture of the sender.

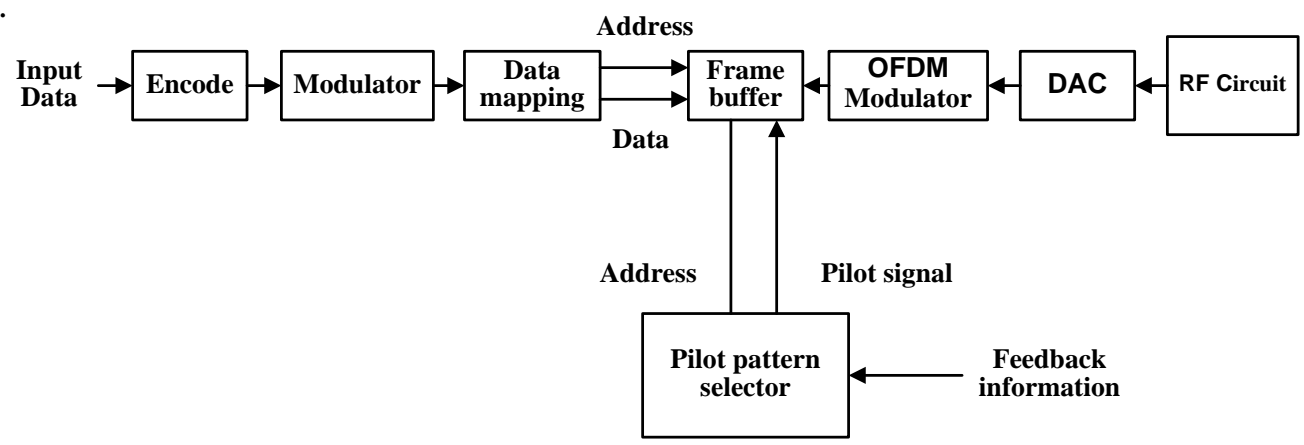

Figure 1. System Architecture of the Sender

The frame buffer of sender will assign its data to the mapping position according to data mapping and the address provided by pilot pattern selector. Thereinto, the pilot pattern selector will receive some feedback information from the receiver, including pilot pattern pointer, multipath number, channel quality information and other important information, which are generated by CS channel parameter estimator of the receiver.

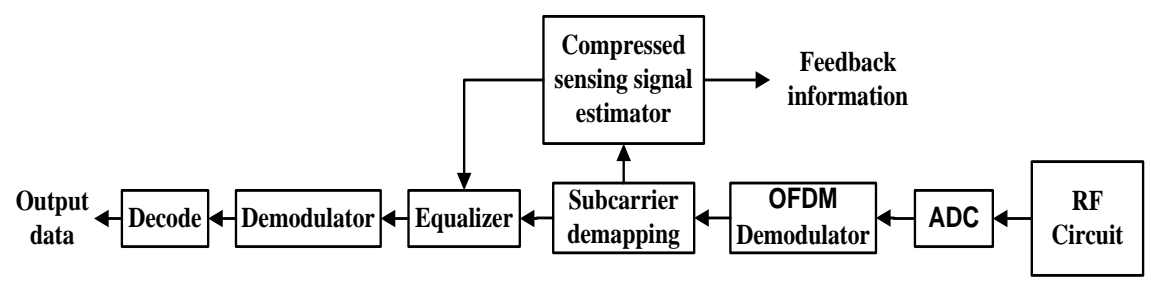

Figure 2. System Architecture of the Receiver

Figure 2 shows system architecture of the receiver. The subcarrier demapping will send pilot signal to CS channel parameter estimator to obtain feedback information and channel response after received signal is demodulated by OFDM. The architecture of CS channel parameter estimator is shown in figure 3. 


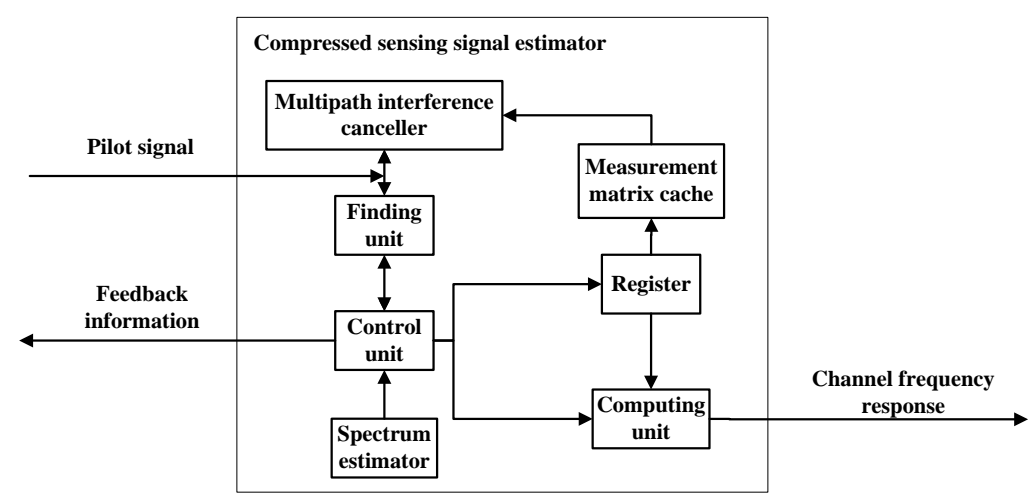

Figure 3. Block Diagram of CS Channel Parameter Estimator

In the second time of CS operation, the multipath interference canceller will use multipath channel influence obained by pilot signal to eliminate the interference. Meanwhile, the finding unit will also search the rest of multipath channels. Thereinto, the finding unit can adopt best matching pursuit or orthogonal matching pursuit and other algorithms. The control unit will determine the times of CS functioning after receiving multipath information from the finding unit, and send the received information to buffer to save. When the multipath channel search is completed, the control unit will rebuild all saved multipath channels to obtain vector $\mathbf{u}$, and pick out a set of pointers from previously configured sparse random pilot style based on parameters such as number of multipath channels and pilot signals etc., and return feedback information to the sender. Then, the computing unit will calculate channel response of current frequency domain to compensate for the equalizer according to vector $\mathbf{u}$ and equation (30). The measurement matrix buffer will calculate the product of measurement matrix $\boldsymbol{\Phi}$ and vector $\mathbf{u}$ and give the result to multipath interference canceller for the next iteration use after receiving buffer information.

\section{Simulation Result}

In the simulation, we first assume that four different kinds of pilot style configuration are arranged in sender and receiver as table 1 shows. Thereinto, 0 and 3 are the upper and lower bound of the number of pilot signals respectively. The initial value of system simulation is 1 .

Table 1. Configuration of Pilot Style

\begin{tabular}{l|l}
\hline pilot pattern index & pilot number \\
\hline P0(Pmin) & 3 \\
\hline P1(Pini) & 6 \\
\hline P2 & 9 \\
\hline P3(Pmax) & 12 \\
\hline
\end{tabular}

The configuration of system simulation parameters is shown in table 2 . 
Table 2. Configuration of System Parameters

\begin{tabular}{|c|c|}
\hline specification & $\begin{array}{l}\text { IEEE 802.11af } \\
(5 \mathrm{MHz})\end{array}$ \\
\hline symbol length & $12.8 \mu \mathrm{s}$ \\
\hline $\begin{array}{l}\text { CP length } \\
(1 / 4) \\
\text { subcarrier spacing }\end{array}$ & $\begin{array}{l}\text { data symbol: } 3.2 \mu \mathrm{s} \\
\text { CE symbol: } 6.4 \mu \mathrm{s} \\
78.125 \mathrm{kHz}\end{array}$ \\
\hline $\begin{array}{l}\text { Pilot Distance } \\
\text { coherent bandwidth } \\
\text { Pilot Distance }<540 \mathrm{kHz}\end{array}$ & $\begin{array}{l}\text { data symbol:78.125 kHz } \\
\text { CE symbol:1.094 MHz } \\
\text { (equivalent to inserting a pilot } \\
\text { into every } 14 \text { subcarriers) } \\
\text { No } \\
\end{array}$ \\
\hline $\begin{array}{l}\text { coherent time } \\
\text { symbol width }<10.88 \mathrm{~ms} \\
\text { (speed: } 60 \mathrm{~km} / \mathrm{hr} \text { ) } \\
\text { channel model }\end{array}$ & ITU Vehicular A (Veh A) \\
\hline channel estimation & $\begin{array}{l}\text { channel parameter estimation } \\
\text { based on compressed sensing }\end{array}$ \\
\hline
\end{tabular}

Figure 4 shows the result of mean square error(MSE). It can be seen that at least six pilot signals are needed in successful application of CS channel parameter estimation and meeting the MSE condition. While the form of sparse random pilot signal can allocate its symbol position through the channel and other related information, making the receiver obtain more accurate channel response to reduce error rate.

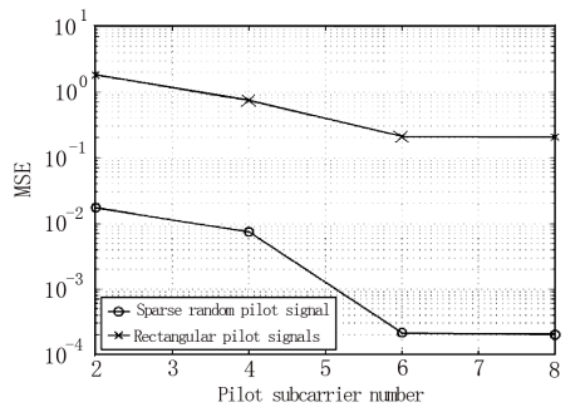

Fig.4 MSE Simulation Result

\section{Conclusion}

This paper mainly applies CS theory to selective wireless channel, using channel's delayed Doppler sparsity to obtain channel coefficient in time-frequency grid. We convert channel estimation into sparse reconstruction, then combine channel estimation with CS theory, which can effectively reduce the use of pilot signal, thereby improving the system spectrum utilization.

Furthermore, the receiver calculates channel information through channel parameter estimator and return feedback information to the sender for the next transmission use. 
While the sender selects suitable polit style and adjusts the position of pilot signal based on the feedback information to match current channel circumstance, thus getting a more accurate channel response and reducing error rate.

\section{Acknowledgements}

This work is supported by "Quality Project" of Degree and postgraduate education in Xi'an Polytechnic University (13YZL05).

\section{References}

[1] E. J. Candés and J. Romberg, "Quantitativerobust uncertainty principles and optimally sparse decompositions", Found. Comput. Math., vol.6, (2006), pp.227-254.

[2] D. Needell and J. A. Tropp, "CoSaMP: Iterative signal recovery from incomplete and inaccurate samples", California Inst. of Technol.,Pasadena, ACM Tech. Rep. (2008).

[3] P. Flandrin, Time-Frequency/Time-Scale Analysis, Academic Press, (1999).

[4] M. Rudelson and R. Vershynin, "Sparse reconstruction by convex relaxation: Fourier and Gaussian measurements", in Proc. 40th Annu. Conf. Inf. Sci. Syst., (2006), pp. 207-212.

[5] H. Rauhut, "Stability results for random sampling of sparse trigonometric polynomials", IEEE Trans. Inf. Theory, vol.54, no.12, (2008), pp.5661-5670.

[6] J. Paredes, G. Arce, and Z. Wang, “ Ultra-wideband compressed sensing: Channel estimation,” IEEE J. Sel. Topics Signal Process., vol.1, no.3, (2007), pp.383-395.

[7] E. Candes and T.Tao, "Near Optimal Signal Recovery from Random projections:Universal encoding strategies", IEEE Trans. Inform. Theory, vol.52, no.12, (2006), pp.5406-5425.

[8] D. Donoho, “Compressed sensing”, IEEE Trans. on Inf. Th., vol.52, (2006), pp.1289-1306.

[9] W. Kozek and A. F.Molisch, "Nonorthogonal pulseshapes for multicarrier communications in doubly dispersive channels", IEEE J. Sel. Areas Comm., vol.16, (1998), pp.1579-1589.

[10] G. Matz, D. Schafhuber, K. Gröchenig, M. Hartmann, and F. Hlawatsch, "Analysis, optimization, and implementation of low-interference wireless multicarrier systems", IEEE Trans. Wireless Comm., vol.6, (2007), pp.1921-1931.

[11] P. A. Bello, "Characterization of randomly time-variant linear channels," IEEE Trans. Comm. Syst., vol.11, (1963), pp.360-393.

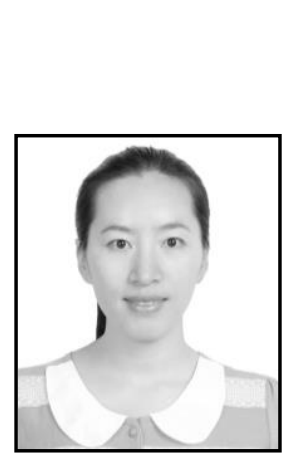

\section{Authors}

Li Li, she is a teacher of Xi'an Polytechnic University, Master, lecturer. She works at college of computer science, and has assumed many projects. The research fields are computer algorithms, parallel computing, intelligent information Processing, etc.

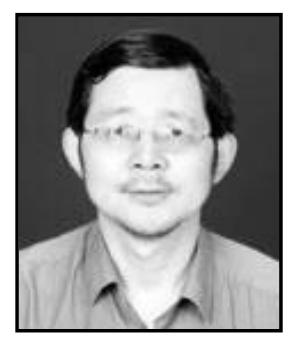

Ning Chen, he is an associated professor of college of computer science, Xi'an polytechnic university in Xi'an, China. His research interests include computer algorithms, parallel computing, intelligent information Processing, etc. 
International Journal of Future Generation Communication and Networking Vol. 8, No. 3 (2015) 